\section{Epitope Based Vaccine Designing- A mini review}

\author{
Aryandra Arya and Sunil K Arora* \\ Department of Immunopathology, Post Graduate Institute of Medical Education \& Research, \\ Chandigarh-160 012, India
}

Received: 09 November, 2020

Accepted: 23 November, 2020

Published: 24 November, 2020

*Corresponding author: Sunil K Arora, Department of Immunopathology, Post Graduate Institute of Medical Education \& Research, Chandigarh-160 012, India, E-mail: arora.sunilkumar@pgimer.edu.in

https://www.peertechz.com

\section{Introduction}

A vaccine is an antigen derived from pathogen. In its crudest form vaccine contains either attenuated pathogen, or an antigen molecule as in case of subunit vaccine, yet what interacts with immune system are few amino acids in the form of epitopes [1]. The idea to form a vaccine from selective few epitopes has emerged as a more logical approach owing to the fact that the conventional approaches are slow and selection of antigen is more or less random. In the last 5 years a lot of new vaccine candidates have been proposed which are based on B Cell Epitopes (BCE) and T Cell Epitopes (TCE) [2]. This approach of rapid identification of immuno epitopes is centered on computational predictions, which utilize advance algorithms and increasing epitope data base. Epitope prediction is one of the most important corner stones of in-silico vaccine designing, however it depends on antigen identification, and most crucially epitope selection for an effective immune response.

The in-silico vaccine designing is known as computational vaccinology. The advantage of computational vaccinology is, utilization of high through put data analysis methods for rapid antigen identification, molecular docking and simulation models to test immunological responses [3]. This method can analyze multiple antigen candidates and whole proteomes for antigenicity and efficacy in a relatively short time. Epitope search has an additional advantage to further narrow down the antigen screening for very short specific regions, thereby providing a possibility where protein-based manipulation can be used to synergies and select the appropriate immune response type (Figure 1 ).

\section{Identification of antigen and computational vaccinology}

With the advent of high through put proteomics, vaccinologists now have access to multiple tools which can analyze a protein sequence for identification and functioning in an organism along with its interaction and possible evolutionary conservation [4-7]. This approach is helpful in providing novel vaccine candidates in a relatively shorter time. One of the main reasons for the failure of a vaccine is its inability to generate specific immune response. By identifying and selecting the most potent antigens, this shortcoming can be avoided. In a recent attempt for a vaccine candidate for visceral leishmaniasis, the authors have applied extensive immuneinformatics approach to identify the most potent antigens based on KEGG (Kyoto Encyclopedia of Genes and Genomes] analysis of proteins involved in Protein-Protein Interaction (PPI)) networks and metabolic pathways $[8,9]$. VaxiJen 2.0 is an alignment independent antigen prediction server, based on physico chemical properties of protein sequences, utilizing Auto Cross Covariance (ACC) $[10,11]$. Another online serve JennerPredict predicts antigen from functional domains of proteins involved in host pathogen interaction [12]. Similarly VacSol can predict potential therapeutic targets using subtractive reverse vaccinology [13]. AntigenDB is a database of previously validated antigens, the dataset includes data from various other databases like Swiss-Prot, MHCBN, AntiJen, IEDB, and BCIPEP [14]. These tools can help in identification of most probable antigens which are able to generate desired immune response.

\section{Epitope mapping and selection}

B-cells recognize discontinuous conformational epitopes and continuous linear epitopes [15-17]. BCE arise due to protein folding as these epitopes are recognized by antibodies. The role of hydrophobic vs hydrophilic regions is open to discussion as it is now known that surface regions of protein contain same number of hydrophilic and hydrophobic residues [18]. Amino acid propensity scales applicable for B-cell epitope prediction are generally based on flexibility [19], $\beta$-turn propensity [20], and surface accessibility [21]. Prediction of 3D conformational epitope is more difficult than $\mathrm{T}$ cell epitopes owing to the 


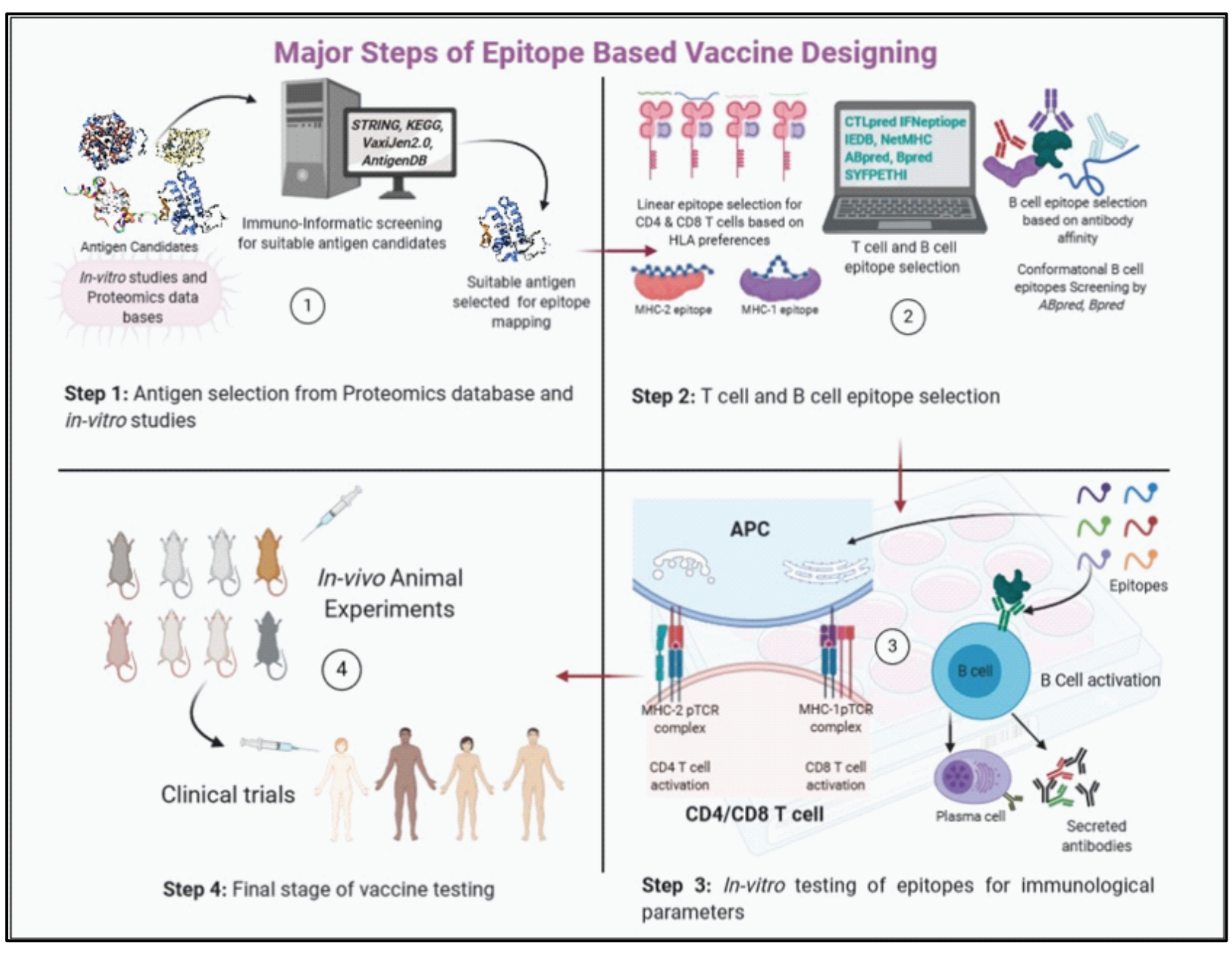

Figure 1: Major steps of epitope-based vaccine designing.

uncertainty in prediction models of protein folding. The existing prediction models of conformational B cell epitopes require antigen 3D structure or homology-based model of the amino acid sequence. So far, no method is available which can predict conformational $\mathrm{B}$ cell epitope using antigen primary sequence in the absence of any homology with the known structures. The conformational B cell epitopes tend to be longer than 17 amino-acid (aa) sequence, since shorter aa sequences generally do not form conformational epitopes. ABCPred and BCPRED are B cell epitope prediction web servers which are based on ANN (Artificial Neural Network) and SVM (Support Vector Machine) $[22,23]$. Bcepred, predict B-cell epitopes on the basis of the physico-chemical properties (hydrophilicity, flexibility/mobility, accessibility, polarity, exposed surface and turns) [24]. The accuracy for ABCPred and Bcepred is $65 \%$ and $58 \%$ respectively. One of the major constraints of accurate B cell epitope prediction is the small size of dataset used for model training, and use of random peptides as non $\mathrm{B}$ cell epitopes. CBtope is another webserver which can predict conformational B cell epitopes using SVM mechanics up to a prediction accuracy of more than $85 \%$ and Area Under Curve (AUC) 0.9 [25]. LBtope is a linear B cell epitope prediction model which is based on SVM and uses a larger dataset of validated B-cell epitopes and non-epitopes (12063 epitopes and 20589 non epitopes obtained from IEDB database) [26].
On the other hand, a T-cell recognizes epitopes in context of MHC-I and MHC-II molecules. The MHC-II molecules are presented on the surface of professional Antigen Presenting Cells (APC), these cells process and present antigens to $\mathrm{CD} 44^{+}$ $\mathrm{T}$-cells through exogenous pathway. The MHC-I is expressed on the surface of all nucleated cells, it presents endogenous antigens (peptides generally associated with cancer and infecting viruses) to $\mathrm{CD} 8+\mathrm{T}$-cells [27]. The peptide length for MHC-I associated epitopes are 9-15 amino acids long, while for MHC-II it can vary up to 22 amino acids. The huge diversity of MHC alleles and T-cell receptors (TCR) presents a challenge in prediction of TSE along with antigen processing during which large protein molecules are chopped into smaller peptides and loaded onto MHC molecule [28]. A large number of prediction software have come up in last 20 years with prediction accuracy levels up to $75 \%$. NetMHCCcons 1.1 has integrated three prediction tools NetMHC, NetMHCPan and PickPocket for more accurate prediction than its competitors [29]. NetChop can predict the proteasomal cleavage sites for MHC-1 epitopes, while TAPPred can predict the binding affinity towards TAP (Transporter associated with antigen processing) $[30,31]$. The TCE prediction software are basically divided in two groups viz. for MHC-I binders and for MHC-II binders. The large number of alleles and sub alleles make it difficult 
to optimize the epitope selection, and for that purpose the Allelefreq.Net software can be used to narrow down the allele requirements based on population selection criteria. Prediction of epitopes for MHC-II binders is comparatively more difficult than for MHC-I owing to two factors: a) the large number of alleles and sub allele frequency in MHC-II loci, and b) due to the physiochemical property of MHC-II grooves, which are open ended and can fit a larger peptide molecule. For MHC-I and MHC-II epitope selection a number of web servers have emerged due to a bigger data sets available for machine learning, majority of servers are based on ANN, SVM, and HMM (Hidden Markov model). SYFPEITHI is an online server, which is based on previous publications of T-cell epitopes and MHC ligands [32]. Similarly, NET MHC-I and NET MHC-II are able to predict TCE for humans as well as mouse [33]. The Propred is an MHC-II prediction server based on quantitative matrix [34].

For a long time, the idea of TCE prediction has been in context of either $\mathrm{CD} 4+\mathrm{T}$-cell or $\mathrm{CD} 8+\mathrm{T}$-cell activation, but none of the methods can predict the nature of $\mathrm{T}$-cell response. The T-cells, after activation may generate different type of responses e.g. either Th1 or Th2. The signature cytokine for Th1 is IFN- $\gamma$ and for Th2 is IL-4, IL-5 and IL-10 [35]. Th1 type of responses are important in context of intracellular infections like viral, parasitic or bacterial infections. The nature of this response depends on multiple different factors like secondary signal for activation, TLR and PAMPS activation, but so far it has not been shown to be dependent on the nature of epitope, although the alteration of single amino acid in epitope has been shown to completely alter the immune response type $[36,37]$. In this context so far, it has been impossible to predict the epitopes for Th1 or Th2 cell responses. One online server, the IFNepiotpe can predict IFN- $\gamma$ inducing epitopes, thereby predicting the immunological response type [38]. Similar to IFNepiotpe CTLpred is based on direct methods of prediction where the information or patterns of $\mathrm{T}$ cell epitopes, instead of MHC binders, are used for the development of methods [39]. The method is based on Artificial Neural network and support vector machine, which allows the consensus and combined prediction based on these two approaches.

\section{Effective vaccine}

In natural course of infection both cell-mediated immunity as well as humoral immunity are required to clear the infection, therefore an integral approach, combining both T-cell and B-cell epitopes, is the appropriate way to design a vaccine. The BCE and TCE can be linked by linker sequences which are amino acids with neutral charge and maximum rotational degree of freedom. Another possibilty to combine TCE and $\mathrm{BCE}$ is to isolate those regions of antigens which are both $\mathrm{B}$ cell epitope positive and $\mathrm{T}$ cell epitope positive. For designing an epitope-based vaccine one must address the question of, how many epitopes and which epitopes to choose. There is no straight forward solution to this problem but it is evident from previous research that large size molecules generate better immune response as they are able to mimic the natural antigen and its course of immune activation, shorter peptides have high Expect value (e value) and the probability of finding similar peptides as self-antigen is relatively larger compared to proteins with larger size $[40,41]$. One possibilty is to take it one step further in this regard by integrating multiple epitopes from different antigens and use them in a cocktail/chimeric manner forming a multiepitope construct to synergize the immune response of single epitope into a cumulative effect. One of the advantages of multiple epitope utilization is the increase in the HLA diversity. More research is required to understand the synergy of epitope integration as very few vaccine candidates with chimeric epitopes have been tested as on today $[42,43]$.

Another significant aspect of vaccine development is the generation of memory response, since no vaccine can be effective if memory response is inadequate to respond to reinfection in a heightened manner. The memory response is linked to two different but associated phenomenon, firstly the TCR signal strength and pro-survival signals received, secondly the influence of cytokines and costimulatory signals on the transcriptomal regulation of $\mathrm{T}$ cell differentiation. The stability of $\mathrm{CD} 4$ + and CD8 + T memory cells generated differs remarkably in their need for cytokine milieu, for example memory CD8+ $\mathrm{T}$-cells proliferate in response to $\mathrm{IL}-15$ but $\mathrm{CD} 4+\mathrm{T}$-cells do not [44]. The generation of memory is linked to the amount and duration for which the antigen persists. Longer exposures of high antigen amount are known to induce senescence in CD8+ T-cells but might be required for CD4+ T-cells $[45,46]$. Memory T-Cells have Stem cell like capabilities and it appears that homeostatic signals drive self-renewal whereas antigenic signals drive effector differentiation. The formation of effector CD8 + T-cell seems to follow a developmental program, which can be triggered by a brief 2-24 hr antigen stimulation and seems to be affected largely by the extrinsic factors such as antigen exposure, its duration and cytokine milieu. Memory T-cells, both $\mathrm{CD} 8+$ and $\mathrm{CD}_{4+}+$, follow one common pattern i.e. they go through an expansion phase upon antigen stimulation, this expansion phase develops effector memory cell and persists for a relatively shorter duration. At the end of effector expansion phase, a contraction phase kills all the effector $\mathrm{T}$ cells, at this stage some effector $\mathrm{T}$ cells survive and change into memory subset. The minimum number of memory $\mathrm{T}$-cells surviving has direct correlation with effective protection against reinfection. Memory T cell number below a certain threshold is ineffective against future reinfection [47]. A vaccine candidate should be able to generate above threshold level Memory $\mathrm{T}$-cells in order to be effective. The initial expansion phase of effector T-cell formation is directly related to the size of memory T-cell at the end $[47,48]$. In an experiment by Murli Kaza et. al. 1998 only $5 \%$ of initially activated CD8+ T cells were able to pass into memory pool, in the same set of experiments the memory pool size for CD8+ T cells was $10 \%$ of total CD8+ $\mathrm{T}$-cell population [49]. Therefore, it is expected from a vaccine candidate to induce a larger effector $\mathrm{T}$-cell population possible. Epitope based vaccines can enhance this initial effector burst phase by utilizing only the relevant and immunogenic epitopes. Another advantage of using epitope-based vaccine compared to conventional vaccines is in case of chronically ill patiens, which have consistent high antigen levels causing $\mathrm{T}$ cell exhaustion. In those cases, epitope identification and use 
of newer or protective epitopes can provide a better vaccine candidate. Epitope based vaccine development provides a better grip on the amount and specificity of antigen required to activate $\mathrm{T}$ cells and can provide better candidates which are effective in generating either Memory CD4+ T cells by using selective MHC-II epitopes or Memory CD8+ T cells by MHC-I epitopes.

\section{Recent developments}

Most important implication of epitope-based vaccines would be to address diseases for which conventional methodologies of vaccine development have been unsuccessful till date, like HIV, TB, Leishmaniasis and SARS COV-2. The designing of a vaccine depends upon the detailed knowledge of natural immune profile of the infection and precise identification of the immune-correlates of protection, as any random antigen may not provide perfect protection. The immune response generated by an antigen depends upon its interaction with TCR, PAMPS and most importantly the HLA. Not every antigen carries the epitopes which can be presented by HLA molecules to a TCR to generate a robust immune response. On the same lines, it is necessary that a strong association between HLA epitope and TCR complex will generate the immune response in a specific manner (Th1 or Th2). The strength and duration of such TCR MHC complexes along with secondary signaling molecules forming the supramolecular activation cluster or SMAC affects the future fate of $T$ cell. Naïve $T$ cells subjected to strong TCR stimulations favor Th1 lineage, while weak signals favor Th2 type [50,51]. Remarkably weak TCR stimulation at immuno synapse is enough to generate a robust memory response, while sustained duration of signal along with higher antigen levels generates enhanced proliferation [52]. The role of epitope alone during TCR and HLA association dictating the downstream events would need to be explored, as this will answer the question why certain antigens are able to generate good memory response and others fail.

Epitope based vaccines are also emerging for cancer therapy and many candidates are being tested on both therapeutic and prophylactic basis. Since almost all proteins from a cancerous cell are similar to the normal proteins present in the body, the minute differences limit to only a few amino acid alterations, which can be utilized as epitope-based vaccine candidate. One candidate to use multiple epitopes is Survivin derived multiepitope vaccine EMD640744 for advanced solid tumors [53]. Another candidate based on anti-FSTL1 [Follistatin-related protein 1] mAbs protein by Kudo and Kawami has shown remarkable immune response against tumors [54].

In the recent outbreak of severe acute respiratory syndromecorona virus-2 [SARS-COV-2], many vaccine candidates have emerged based on epitope mapping. Hong-Zhi Chen et al. 2020, have identified BCE and TCE from nucleocapsid protein of SARS-COV-2 virus as probable candidates for vaccine designing [55]. The epitopes are based on ABCpred and BepiPred servers for sequential B-cell epitope selection and discontinuous B-cell epitopes identification by DiscoTope 2.0 [56]. In the same study IEDB server has been utilized for HLA-I and HLA-II binding peptides computation. Tamalika
Kar, et al. have proposed a multi-epitope vaccine using spike glycoprotein of SARS-CoV-2, docking of vaccine candidate confirmed stable interactions with TLRs and MHC [57]. For all the proposed vaccines for SARS-CoV-2 the immune-efficacy has been assessed in-silico by immune-simulation. Many of these candidates are hypothetical and are not validated by in-vitro analysis for immunological responses instead they have utilized molecular docking to give an early assessment. The vaccine designing for SARS-COV-2 has emphasized the importance of immune-informatics and epitope mapping to identify protein regions, which are physiologically vital for the virus and have the ability to generate immune response.

\section{Concluding remarks}

With ever increasing database of confirmed epitopes and new algorithms for computation, $\mathrm{B}$ and $\mathrm{T}$ cell epitope prediction is becoming more reliable for novel vaccine designing. After the emergence of servers like IFNepitope, it might become possible in future to identify cytokine specific epitopes . These cytokine specific epitopes could then be used as therapeutic candidates. The presence of cytokine specific epitopes could be the reason why crude antigens fail to generate a specific immune response (presence of undesirable epitopes), although there is no experimental evidence present and this idea requires more research. Nonetheless, the possibility of cytokine specific epitopes has not only provided us a tool for vaccine designing but has also emphasized on the possible role of epitope alone in $\mathrm{T}$ cell activation. This question seems to be centered around the role of $\mathrm{T}$ cell receptor and its mechanism of activation. Currently two mechanisms available to address this scenario are, Conformation Change Model and the Kinetic Segregation Model, both models fail to address how a single amino acid substitution in the epitope can results in different downstream pathway activation. Since it is an epitope, which actually holds the specificity while generating an antibody response and $\mathrm{T}$ cell activation, the non-epitope domains of protein become not only useless but can also sterically shield the immunogenic epitope domains. Thus, the epitope-based vaccine designing can provide us with new candidates in this regard where large antigen derived vaccines have not been successful.

\section{References}

1. Hwang JR, Byeon Y, Kim D, Park SG (2020) Recent insights of T cell receptormediated signaling pathways for T cell activation and development. Exp Mol Med 52: 750-761. Link: https://bit.ly/396vks9

2. Palatnik-de-Sousa CB, Soares I da S, Rosa DS (2018) Editorial: Epitope Discovery and Synthetic Vaccine Design. Front Immunol 9. Link: https://bit.ly/338fLMz

3. Folcik VA, An GC, Orosz CG (2007) The Basic Immune Simulator: An agent based model to study the interactions between innate and adaptive immunity. Theor Biol Med Model 4: 39. Link: https://bit.ly/2KzLb8q

4. Kanehisa M, Goto S (2000) KEGG: Kyoto Encyclopedia of Genes and Genomes Nucleic Acids Res 28: 27-30. Link: https://bit.ly/3nWPqtb

5. Kumar S, Nei M, Dudley J, Tamura K (2008) MEGA: A biologist-centric software for evolutionary analysis of DNA and protein sequences. Brief Bioinform 9: 299-306. Link: https://bit.ly/339gq08 
6. Waterhouse A, Bertoni M, Bienert S, Studer G, Tauriello G, et al. (2018) SWISSMODEL: homology modelling of protein structures and complexes. Nucleic Acids Res 46: W296-W303. Link: https://bit.ly/3nLCPZq

7. Szklarczyk D, Gable AL, Lyon D, Junge A, Wyder S, et al. (2019) STRING v11: protein-protein association networks with increased coverage, supporting functional discovery in genome-wide experimental datasets. Nucleic Acids Res 47: D607- D13.

8. Brito RCF, Guimarães FG, Velloso JPL, Corrêa-Oliveira R, Ruiz JC, et al. (2017) Immunoinformatics Features Linked to Leishmania Vaccine Development Data Integration of Experimental and In Silico Studies. Int J Mol Sci 18. Link: https://bit.ly/3nQT6MV

9. De Brito RCF, Cardoso JM de O, Reis LES, Mathias FAS, Aguiar-Soares RD de O, et al. (2019) Synthetic Peptides Elicit Strong Cellular Immunity in Visceral Leishmaniasis Natural Reservoir and Contribute to Long-Lasting Polyfunctional T-Cells in BALB/c Mice. Vaccines 7. Link: https://bit.ly/2J4UpbU

10. Doytchinova IA, Flower DR (2007) VaxiJen: a server for prediction of protective antigens, tumour antigens and subunit vaccines. BMC Bioinformatics 8: 4 Link: https://bit.ly/2J9AJDF

11. Nyström $\AA$, Andersson PM, Lundstedt T (2000) Multivariate Data Analysis of Topographically Modified a-Melanotropin Analogues using Auto and Cross Auto Covariances (ACC). Quant Struct-Act Relatsh 19: 264-269. Link: https://bit.ly/2HxprsE

12. Jaiswal V, Chanumolu SK, Gupta A, Chauhan RS, Rout C (2013) Jennerpredict server: prediction of protein vaccine candidates (PVCs) in bacteria based on host-pathogen interactions. BMC Bioinformatics 14: 211. Link: https://bit.ly/3mbWbXe

13. Rizwan M, Naz A, Ahmad J, Naz K, Obaid A, et al. (2017) VacSol: a high throughput in silico pipeline to predict potential therapeutic targets in prokaryotic pathogens using subtractive reverse vaccinology. BMC Bioinformatics 18. Link: https://bit.ly/3pPJ6p3

14. Ansari HR, Flower DR, Raghava GPS (2010) AntigenDB: an immunoinformatics database of pathogen antigens. Nucleic Acids Res 38: D847- D53. Link: https://bit.ly/370qMkp

15. Langeveld JPM, Martinez-Torrecuadrada J, Boshuizen RS, Meloen RH, Ignacio Casal J (2001) Characterisation of a protective linear B cell epitope against feline parvoviruses. Vaccine 19: 2352-2360. Link: https://bit.ly/3I7xNVL

16. Walter $\mathrm{G}$ (1986) Production and use of antibodies against synthetic peptides. J Immunol Methods 88: 149-161. Link: https://bit.ly/3IUz7MG

17. Barlow DJ, Edwards MS, Thornton JM (1986) Continuous and discontinuous protein antigenic determinants. Nature 322: 747-748. Link: https://go.nature.com/3pZcERg

18. Lins L, Thomas A, Brasseur R (2003) Analysis of accessible surface of residues in proteins. Protein Sci 12: 1406-1417. Link: https://bit.ly/3pP2TEW

19. Karplus PA, Schulz GE (1985) Prediction of chain flexibility in proteins. Naturwissenschaften 72: 212-213. Link: https://bit.ly/2J9zggD

20. Pellequer JL, Westhof E, Van Regenmortel MHV (1993) Correlation between the location of antigenic sites and the prediction of turns in proteins. Immuno Lett 36: 83-99. Link: https://bit.ly/3pRbcjP

21. Emini EA, Hughes JV, Perlow DS, Boger J (1985) Induction of hepatitis A virusneutralizing antibody by a virus-specific synthetic peptide. J Virol 55: 836-839. Link: https://bit.ly/3nRyKTA

22. Saha S, Raghava GPS (2006) Prediction of continuous B-cell epitopes in an antigen using recurrent neural network. Proteins 65: 40-48. Link: https://bit.ly/36VvcsA

23. EL-Manzalawy Y, Dobbs D, Honavar V (2008) Predicting linear B-cell epitopes using string kernels. J Mol Recognit 21: 243-255. Link: https://bit.ly/3kV2kWF
24. Saha S, Raghava GPS (2004) BcePred: Prediction of Continuous B-Cell Epitopes in Antigenic Sequences Using Physico-chemical Properties. In Nicosia G, Cutello V, Bentley PJ, Timmis J, editors. Artificial Immune Systems. Berlin, Heidelberg: Springer 197-204. (Lecture Notes in Computer Science).

25. Ansari HR, Raghava GP (2010) Identification of conformational B-cell Epitopes in an antigen from its primary sequence. Immunome Res 6: 6. Link: https://bit.ly/2J6nOlU

26. Singh H, Ansari HR, Raghava GPS (2013) Improved method for linear B-cell epitope prediction using antigen's primary sequence. PloS One 8: e62216. Link: https://bit.ly/3ftg2Pe

27. Blum JS, Wearsch PA, Cresswell P (2013) Pathways of Antigen Processing Annu Rev Immunol 31: 443-473. Link: https://bit.ly/2KpQgQr

28. Robinson JH, Delvig AA (2002) Diversity in MHC class II antigen presentation. Immunology 105: 252-262. Link: https://bit.ly/3kTgs2w

29. Karosiene E, Lundegaard C, Lund O, Nielsen M (2012) NetMHCcons: a consensus method for the major histocompatibility complex class I predictions. Immunogenetics 64: 177-186. Link: https://bit.ly/2ITDw4p

30. Nielsen M, Lundegaard C, Lund O, Keşmir C (2005) The role of the proteasome in generating cytotoxic T-cell epitopes: insights obtained from improved predictions of proteasomal cleavage. Immunogenetics 57: 33-41. Link: https://bit.ly/3pUYobT

31. Bhasin M, Lata S, Raghava GPS (2007) TAPPred prediction of TAPbinding peptides in antigens. Methods Mol Biol 409: 381-396. Link: https://bit.ly/3kZKykM

32. Rammensee HG, Bachmann J, Emmerich NPN, Bachor OA, Stevanović S (1999) SYFPEITHI: database for MHC ligands and peptide motifs. Immunogenetics 50: 213-219. Link: https://bit.ly/3nRFSiV

33. Lundegaard C, Lamberth K, Harndahl M, Buus S, Lund O, et al. (2008) NetMHC-3.0: accurate web accessible predictions of human, mouse and monkey MHC class I affinities for peptides of length 8-11. Nucleic Acids Res 36: W509- W512. Link: https://bit.ly/2UU5Ju9

34. Singh H, Raghava GP (2001) ProPred: prediction of HLA-DR binding sites Bioinformatics 17: 1236-1237. Link: https://bit.ly/37259jx

35. Constant SL, Bottomly K (1997) Induction of Th1 and Th2 CD4+ T cell responses: the alternative approaches. Annu Rev Immunol 15: 297-322. Link: https://bit.ly/33c123i

36. Pfeiffer C, Stein J, Southwood S, Ketelaar H, Sette A, et al. (1995) Altered peptide ligands can control CD4 T lymphocyte differentiation in vivo. J Exp Med 181: 1569-1574. Link: https://bit.ly/33bjkSs

37. Windhagen A, Scholz C, Höllsberg P, Fukaura H, Sette A, et al. (1995) Modulation of cytokine patterns of human autoreactive $T$ cell clones by a single amino acid substitution of their peptide ligand. Immunity 2: 373-380. Link: https://bit.ly/3nRmliM

38. Dhanda SK, Vir P, Raghava GPS (2013) Designing of interferon-gamma inducing MHC class-II binders. Biol Direct 8: 30. Link: https://bit.ly/36ZUeqN

39. Bhasin M, Raghava GPS (2004) Prediction of CTL epitopes using QM, SVM and ANN techniques. Vaccine 22: 3195-3204. Link: https://bit.ly/39b7iMM

40. Zhao C, Sun Y, Zhao Y, Wang S, Yu T, et al. (2012) Immunogenicity of a multiepitope DNA vaccine against hantavirus. Hum Vaccines Immunother 8: 208215. Link: https://bit.ly/3nRFilf

41. Depla E, Aa AV der, Livingston BD, Crimi C, Allosery K, et al. (2008) Rational Design of a Multiepitope Vaccine Encoding T-Lymphocyte Epitopes for Treatment of Chronic Hepatitis B Virus Infections. J Virol 82: 435-450. Link: https://bit.ly/2HshIMi

42. Rostamtabar M, Rahmani A, Baee M, Karkhah A, Prajapati VK, et al. (2019) 
Development a multi-epitope driven subunit vaccine for immune response reinforcement against Serogroup B of Neisseria meningitidis using comprehensive immunoinformatics approaches. Infect Genet Evol 75: 103992. Link: https://bit.ly/2UUqLc7

43. Tian L, Wang $\mathrm{H}$, Lu D, Zhang $\mathrm{Y}$, Wang $\mathrm{T}$, et al. (2008) The immunoreactivity of chimeric multi-epitope DNA vaccine against IBV in chickens. Biochem Biophys Res Commun 377: 221-225. Link: https://bit.ly/33aowWL

44. Zhang X, Sun S, Hwang I, Tough DF, Sprent J (1998) Potent and selective stimulation of memory-phenotype CD8+ T cells in vivo by IL-15. Immunity 8 : 591-599. Link: https://bit.ly/35VTX90

45. Edwards BH, Bansal A, Sabbaj S, Bakari J, Mulligan MJ, Goepfert PA (2002) Magnitude of functional CD8+ T-cell responses to the gag protein of human immunodeficiency virus type 1 correlates inversely with viral load in plasma. $J$ Virol 76: 2298-2305. Link: https://bit.ly/2Hxm70E

46. Iezzi G, Karjalainen K, Lanzavecchia A (1998) The duration of antigenic stimulation determines the fate of naive and effector T cells. Immunity 8: 8995. Link: https://bit.ly/3IYxg9P

47. Kaech SM, Wherry EJ, Ahmed R (2002) Effector and memory T-cell differentiation: implications for vaccine development. Nat Rev Immunol 2: 251-262. Link: https://bit.ly/33coi1d

48. Busch DH, Pilip IM, Vijh S, Pamer EG (1998) Coordinate regulation of complex $T$ cell populations responding to bacterial infection. Immunity 8: 353-362. Link: https://bit.ly/2J5BA8E

49. Murali-Krishna K, Altman JD, Suresh M, Sourdive DJ, Zajac AJ, et al. (1998) Counting antigen-specific CD8 T cells: a reevaluation of bystander activation during viral infection. Immunity 8: 177-187. Link: https://bit.ly/3fqzgF6
50. Brogdon JL, Leitenberg D, Bottomly K (2002) The potency of TCR signaling differentially regulates NFATc/p activity and early IL-4 transcription in naive CD4+ T cells. J Immunol 168: 3825-3832. Link: https://bit.ly/2UVONFo

51. Constant S, Pfeiffer C, Woodard A, Pasqualini T, Bottomly K (1995) Extent of $T$ cell receptor ligation can determine the functional differentiation of naive CD4+ T cells. J Exp Med 182: 1591-1596. Link: https://bit.ly/3991TFL

52. Daniels MA, Teixeiro E (2015) TCR Signaling in T Cell Memory. Front Immunol. 6: 617. Link: https://bit.ly/3pY8ysg

53. Lennerz V, Gross S, Gallerani E, Sessa C, Mach N, et al. (2014) Immunologic response to the survivin-derived multi-epitope vaccine EMD640744 in patients with advanced solid tumors. Cancer Immunol Immunother CII 63: 381-394. Link: https://bit.ly/3fub8Sk

54. Kudo-Saito C, Ishida A, Shouya Y, Teramoto K, Igarashi T, et al. (2018) Blocking the FSTL1-DIP2A Axis Improves Anti-tumor Immunity. Cell Rep 24: 1790-1801. Link: https://bit.ly/375XdOr

55. Chen HZ, Tang LL, Yu XL, Zhou J, Chang YF, et al. (2020) Bioinformatics analysis of epitope-based vaccine design against the novel SARS-CoV-2. Infect Dis Poverty 9: 88. Link: https://bit.ly/2J2vCW6

56. Kringelum JV, Lundegaard C, Lund O, Nielsen M (2012) Reliable B Cell Epitope Predictions: Impacts of Method Development and Improved Benchmarking PLOS Comput Biol 8: e1002829. Link: https://bit.ly/3kWM0ok

57. Kar T, Narsaria U, Basak S, Deb D, Castiglione F, et al. (2020) A candidate multi-epitope vaccine against SARS-CoV-2. Sci Rep 10: 10895. Link: https://go.nature.com/3pUa0Mv

\section{Discover a bigger Impact and Visibility of your article publication with}

\section{Peertechz Publications}

\section{Highlights}

* Signatory publisher of ORCID

* Signatory Publisher of DORA (San Francisco Declaration on Research Assessment)

- Articles archived in worlds' renowned service providers such as Portico, CNKI, AGRIS, TDNet, Base (Bielefeld University Library), CrossRef, Scilit, J-Gate etc.

* Journals indexed in ICMJE, SHERPA/ROMEO, Google Scholar etc.

* OAI-PMH (Open Archives Initiative Protocol for Metadata Harvesting)

* Dedicated Editorial Board for every journa

* Accurate and rapid peer-review process

- Increased citations of published articles through promotions

* Reduced timeline for article publication

Submit your articles and experience a new surge in publication services (https://www.peertechz.com/submission).

Peertechz journals wishes everlasting success in your every endeavours.

Copyright: () 2020 Arya A, et al. This is an open-access article distributed under the terms of the Creative Commons Attribution License, which permits unrestricted use, distribution, and reproduction in any medium, provided the original author and source are credited. 